\title{
MHC class I allele diversity in cynomolgus macaques of Vietnamese origin
}

\author{
Shuting Huang ${ }^{\text {Equal first author, } 1}$, Xia Huang ${ }^{\text {Equal first author, } 1}{ }^{1}$, Shuang $\mathbf{L i}^{1}$, Mingjun Zhu $^{1}$, Min Zhuo $^{\text {Corresp. } 1}$ \\ ${ }^{1}$ School of Biology and Biological Engineering, South China University of Technology, Guangzhou, Guangdong, China \\ Corresponding Author: Min Zhuo \\ Email address: zhuomin@scut.edu.cn
}

Cynomolgus macaques (Macaca fascicularis, Mafa) have been used as important experimental animal models for carrying out biomedical researches. The results of biomedical experiments strongly depend on the immunogenetic background of animals, especially on the diversity of major histocompatibility complex (MHC) alleles. However, there is much less information available on the polymorphism of MHC class I genes in cynomolgus macaques, than is currently available for humans. In this study, we have identified $40 \mathrm{Mafa}-A$ and $60 \mathrm{Mafa}-B$ exons 2 and 3 sequences from 30 unrelated cynomolgus macaques of Vietnamese origin. Among these alleles, 28 are novel. As for the remaining 72 known alleles, 15 alleles are shared with other cynomolgus macaque populations and 32 are identical to alleles previously reported in other macaque species. A potential recombination event was observed between Mafa-A1*091:02 and Mafa$A 1 * 057: 01$. In addition, the Mafa-A1 genes were found to be more diverse than human $H L A-A$ and the functional residues for peptide binding sites (PBS) or TCR binding sites (TBS) in Mafa-A1 have greater variability than that for non-PBS or non-TBS regions. Overall, this study provides important information on the diversity of Mafa- $A$ and Mafa- $B$ alleles from Vietnamese origin, which may help researchers to choose the most appropriate animals for their studies. 
1 Article category : Original Manuscript

2

3 Title : MHC class I allele diversity in cynomolgus macaques of Vietnamese origin

5 Running Head: MHC class I allele diversity in macaques

6

7 Authors :

8 Shuting Huang ${ }^{1, \S}$, Xia Huang ${ }^{1,}$, Shuang $\mathrm{Li}^{1}$, Mingjun $\mathrm{Zhu}^{1}$, Min Zhuo ${ }^{1, *}$

${ }^{1}$ School of Biology and Biological Engineering, South China University of Technology,

11 Guangzhou 510006, China

* Corresponding author.

Phone number: +86-13560121763

Fax:86-20-39380601

E-mail address: zhuomin@scut.edu.cn

$\S$ Shuting Huang and Xia Huang contributed equally to this article. 
22

23

24

25

26

27

28

29

\section{Abstract}

Cynomolgus macaques (Macaca fascicularis, Mafa) have been used as important experimental animal models for carrying out biomedical researches. The results of biomedical experiments strongly depend on the immunogenetic background of animals, especially on the diversity of major histocompatibility complex (MHC) alleles. However, there is much less information available on the polymorphism of MHC class I genes in cynomolgus macaques, than is currently available for humans. In this study, we have identified $40 \mathrm{Mafa}-\mathrm{A}$ and $60 \mathrm{Mafa}-\mathrm{B}$ exons 2 and 3 sequences from 30 unrelated cynomolgus macaques of Vietnamese origin. Among these alleles, 28 are novel. As for the remaining 72 known alleles, 15 alleles are shared with other cynomolgus macaque populations and 32 are identical to alleles previously reported in other macaque species. A potential recombination event was observed between Mafa-A1*091:02 and Mafa-A1*057:01. In addition, the $M a f a-A 1$ genes were found to be more diverse than human $H L A-A$ and the functional residues for peptide binding sites (PBS) or TCR binding sites (TBS) in Mafa-A1 have greater variability than that for non-PBS or non-TBS regions. Overall, this study provides important information on the diversity of $M a f a-A$ and $M a f a-B$ alleles from Vietnamese origin, which may help researchers to choose the most appropriate animals for their studies.

Keywords: diversity; major histocompatibility complex; cynomolgus macaque 


\section{Introduction}

The MHC glycoproteins, usually known as MHC class I and class II molecules, play important roles in the regulation of innate and adaptive immune response. The MHC classical class I molecules contribute both to innate immunity, by engaging Natural Killer (NK) cell receptors, and to adaptive immunity, by presenting antigens to $\mathrm{CD} 8^{+} \mathrm{T}$ cells to induce their activation and cytotoxicity ${ }^{1}$. Correlating with these functions, the antigen-binding sites, which are mostly located throughout the $\alpha 1$ and $\alpha 2$ domains encoded by the exons 2 and 3, exhibit the highest polymorphism within the full length of MHC class I gene sequences. Scholars found that the polymorphism of MHC genes is generated by a combination of mutation, recombination, and gene duplication and loss, and is maintained over time by selection ${ }^{2-4}$. Numerous infectious and autoimmune diseases are strongly associated with particular MHC alleles and haplotypes ${ }^{5-8}$. For example, human susceptibility to rheumatoid arthritis (RA) was found linked strongly with certain MHC class I and II alleles, including $H L A-D R B 1, H L A-D P B 1$ and $H L A-B{ }^{9}$. In response to HIV, human MHC class I genes, $H L A-B * 57$ and $H L A-B * 27$ exhibit strong and consistent association with lower viral loads in the chronic phase and slow disease progression ${ }^{10}$. In contrast, $H L A-B * 35$ and $H L A-B * 58$ associating with rapid disease progression have also been reported ${ }^{5}$. The cynomolgus macaque and the rhesus macaque (Macaca mulatta, Mamu), are both important nonhuman primate animal models for the study of various human diseases such as acquired immunodeficiency syndrome, tuberculosis, Alzheimer's disease, Parkinson's disease, diabetes, as well as transplantation researches and pharmacodynamic evaluation ${ }^{11-17}$. The rhesus monkey model of collagen-induced arthritis (CIA) is widely used to study the pathogenesis of human RA. It was reported that Mamu- ${ }^{*} 001$ is resistant to CIA ${ }^{18}$. The CIA-susceptible rhesus monkeys need to be preselected on the basis of absence of Mamu- ${ }^{*} 001^{19}$. The Indian-origin rhesus macaque of SIV infection is firstly used as an AIDS model. Mamu-A1*001, Mamu$A 3 * 13: 03, M a m u-B * 008$ and $M a m u-B * 017$ are known as protective alleles and macaques possessing these alleles tend to show slow disease progression after SIVmac251/SIVmac239 challenge 5,15,20,21. Of note, several alleles, Mamu- $A 1 * 001$, Mamu-B*001, and Mamu-B*017 are 
distributed at high frequencies ${ }^{22,23}$. Additionally, anchor residues of CTL epitopes presented by Mamu- $B^{*} 017 / M a m u-B^{*} 008$ were indicated to be similar to those restricted by $H L A-B * 57 / H L A$ $B * 27^{24}$. Due to the extensive characterization of several of these alleles, Indian rhesus macaque is the most widely utilized model in AIDS research. Since the export of rhesus macaques from India was restricted in 1978, the use of cynomolgus macaques for biomedical research has become increasingly prevalent. This species inhabits widely throughout Southeast Asia, including the Philippines, Indonesia, Vietnam, Malaysia, Thailand, Cambodia, and Brunei ${ }^{25}$. In addition, it also had been introduced to Mauritius island located in the western Indian Ocean about 400 years ago, where the Mauritius cynomolgus macaque had become an insular population ${ }^{26}$. Previous studies have demonstrated that most MHC class I alleles found in cynomolgus macaques are unique to animals from particular regions. The distribution frequencies of MHC alleles in distinct population are also different ${ }^{27-29}$. For example, the MHC diversity of the Mauritius cynomolgus macaque is more limited than that of other populations ${ }^{30}$. Hence, the information on MHC diversity from different regions as well as their association with various disease susceptibility needs to be considered carefully, when cynomolgus macaques were used in biomedical studies ${ }^{31}$.

The human classical MHC class I genes, $H L A-A, H L A-B$, and $H L A-C$, exhibit high polymorphism. $5018 H L A-A$ and $6096 H L A-B$ alleles have been included in the IMGT/HLA database (Release 3.36.0, 2019-04-17), which is a module of The Immuno Polymorphism Database (IPD) ${ }^{32}$. In comparison, the orthologues of the $H L A-C$ gene have not been identified so far in macaques and the $M H C-A$ and $M H C-B$ genes in macaques have more complex organization than the human genes ${ }^{33-35}$. Only one copy of the $H L A-A$ and $H L A-B$ genes are present in humans, whilst seven A-like genes and up to nine B-like genes are present in macaques. Furthermore, a novel gene locus, Mafa- $A 8^{*} 01: 01$, was discovered recently in cynomolgus macaque of Filipino origin. Both the $M H C-A$ and $M H C-B$ loci are duplicated in cynomolgus macaque during evolution. Nevertheless, only 375 Mamu- $A$ and 513 Mamu-B alleles, along with $494 M a f a-A$ and $717 M a f a-B$ alleles, have been deposited in the IPD-MHC 
93

94

database (Release 3.2.0.0.) ${ }^{34,36}$. In comparison to their human counterparts, the number and the detailed information on the polymorphism of MHC classic class I genes in macaques are still lacking. The cynomolgus macaques bred in South China mainly originated from Vietnam and have been exported to various places in the world for biomedical research ${ }^{37}$. To better understand the characteristics of their MHC class I alleles, the polymorphism analysis of both the $M a f a-A$ and the Mafa-B exons 2 and 3 sequences were carried out simultaneously in 30 unrelated animals.

\section{Materials and methods}

\section{Animals}

All cynomolgus monkeys were housed in the South China Primate Research \& Development Center (Guangdong, China) and were clinically asymptomatic for known diseases. Peripheral blood samples were collected from over 30 unrelated Vietnamese-origin cynomolgus macaques. The experiments were reviewed and approved by the Institutional Animal Care and Use Committee (IACUC) of Guangdong Landau Biotechnology Co. Ltd. (project number: IACUC$003)$.

\section{RNA extraction, cDNA cloning and sequencing of MHC class I genes}

Total RNA was extracted from peripheral blood mononuclear cell samples of 30 animals using E.Z.N.A. ${ }^{\mathrm{TM}}$ Blood RNA Kits (OMEGA bio-tek). cDNA was synthesized using a PrimeScript ${ }^{\mathrm{TM}}$ II $1^{\text {st }}$ Strand cDNA Synthesis Kit (TaKaRa). Amplification of the full length of exons 2 and 3 sequences was investigated using specific primer pairs (Mafa-A: 'A-F' 5'AACCCTCCTCCTGGTGCTCT-3', and 'A-R' 5'-GGAAGGTTCCATCTCCTGCAG-3', MafaB: 'B-F' 5'- AACCCTCCTCCTGCTGCT-3', and 'B-R' 5'- TGGACTGGGAAGATGGCT-3') The two upstream primers are both located in exon 1 and the two downstream primers are located in exon 4. PCR employed a denaturation process for $3 \mathrm{~min}$ at $94{ }^{\circ} \mathrm{C}$, followed by 32 
117 cycles at $94{ }^{\circ} \mathrm{C}$ for $30 \mathrm{~s}, 58^{\circ} \mathrm{C}($ Mafa-A $)$ or $56^{\circ} \mathrm{C}(M a f a-B)$ for $30 \mathrm{~s}, 72^{\circ} \mathrm{C}$ for 1 min, with a final 118 process at $72{ }^{\circ} \mathrm{C}$ for $8 \mathrm{~min}$. Ex Taq DNA polymerase (TaKaRa) was used in this reaction. PCR 119 products were purified and cloned into the pMD18-T vector (TaKaRa). For each animal, about

12030 clones were selected for Mafa- $A$ and $M a f a-B$ respectively and then were sequenced

121 bidirectionally by the service provider (Beijing Genomics Institute, Shenzhen). Nucleotide

122 sequences of cDNAs were assembled and processed using SeqMan (DNASTAR ${ }^{38}$ ) and aligned

123 using the Clustal W program (BioEdit ${ }^{39}$ ). To ensure authenticity, each sequence was uniquely

124 named if three or more identical clones were observed from at least two individuals, or from two

125 independent PCR for an individual. These sequences were then submitted to the GenBank for

126 accession numbers and to the IPD-MHC database for allele nomenclature ${ }^{40,41}$.

127

128

129

130

131

132

133

134

135

136

137

138

139

140

141

\section{Phylogenetic analysis}

Recombination analysis was performed using the Recombination Detection Program version 4 (RDP4; ${ }^{42}$ ) with a window size of 20 nucleotides and P value less than 0.000005 , and using the Recombination Identification Program (RIP) with a window size of 200 and 99\% confidence intervals (http://www.hiv.lanl.gov/) ${ }^{43}$. A phylogenetic tree was constructed using the neighborjoining (NJ) method ${ }^{44}$ in MEGA7 ${ }^{45}$ using exons 2 and 3. Evolutionary distances were computed using the Kimura 2-parameter model ${ }^{46}$ and assessed using 1000 bootstrap replicates. Values greater than $50 \%$ were used as data-points to construct the tree. The nucleotide polymorphic sites were analyzed by DnaSP. The frequency for the second-most common nucleotide at each position was calculated by the number of occurrences of a nucleotide divided by the number of $M a f a-A 1$ sequences used in this analysis. The frequency for the second-most common amino acid at each position was also calculated by the number of occurrences of an amino acid divided by the number of Mafa-Al amino acid sequences used in this analysis ${ }^{2}$.

\section{Results and discussion}

\section{Summary of the identified MHC class I alleles}


142

143

144

145

146

147

148

149

150

151

152

153

154

155

156

157

158

159

160

161

162

163

164

165

166

167

168

cDNA clones were obtained by RT-PCR using $M a f a-A$ and $M a f a-B$ specific primer pairs. A total of 1965 clones were sequenced, and 882 Mafa- $A$ and 859 Mafa-B cDNA sequences were acquired. After sequence alignment and filtering out the sequences detected identical in less than three clones, we identified $100 \mathrm{MHC}$ class I alleles from 30 cynomolgus macaques of Vietnamese origin, including $40 \mathrm{Mafa}-A$ and $60 \mathrm{Mafa}-B$ genes, of which 28 alleles (11 Mafa- $A$, $17 \mathrm{Mafa}-\mathrm{B})$ were identified as new ones. Their allele names, accession numbers, shared alleles in other cynomolgus macaque populations and counterparts in other macaque species are listed in Tables 1 and 2, respectively. Among the 28 novel alleles, 5 of them, namely Mafa-A1*048:01 (KT907348), Mafa-B*006:01:01 (KT895494), Mafa-B*112:01 (KT895480), Mafa-B*180:01 (KT895475) and Mafa- $B^{* 202: 01 ~(K T 895441), ~ w e r e ~ n e w l y ~ d e t e c t e d ~ i n ~ c y n o m o l g u s ~ m a c a q u e . ~}$ The other 23 alleles are new at five- to seven-digit levels of classification. In recent years, it has been found that the epitranscriptomic modifications of mRNA play very important roles in the regulation of gene expression ${ }^{47}$. However, any kind of epitranscriptomic modification on MHC RNAs hasn't been reported currently ${ }^{48}$. Therefore, we need to further study the existence of mRNA modifications in the transcripts of these 28 new alleles in the future. The remaining 72 alleles have been reported previously in the IPD-MHC database, with 41 of them (17 Mafa- $A, 24$ $M a f a-B)$ being identified in our laboratory ${ }^{49-51}$.

Among the 40 Mafa- $A$ alleles, 35 sequences originated from the Mafa-A1 locus, with the other 2, 1, and 2 alleles originating from $M a f a-A 2,-A 3$, and $-A 4$ loci, respectively. This means that most $M a f a-A$ alleles are expressed in the Mafa- $A 1$ locus ${ }^{28,29}$. As for Mafa-B alleles, the locus number designation has not yet been introduced for them because the macaque $M H C-B$ genes greatly differ in number between haplotypes ${ }^{34,41}$. Amongst the 30 animals analyzed, each individual expressed 1 to $5 \mathrm{Mafa}-A$ genes and 2 to $7 \mathrm{Mafa}-\mathrm{B}$ genes. On average each monkey expressed 6.8 $M a f a-A /-B$ genes. Especially, 11 animals were found to express more than 2 Mafa- $A$ sequences. And 14 macaques were found to have more than 4 Mafa-B alleles. These data showed that both $M H C-A$ and $-B$, especially $-B$ genes, were duplicated in cynomolgus macaque of Vietnamese origin. Many of the macaques may contain at least two Mafa- $A$ and three Mafa-B genes loci. 
169 This is similar to the finding of previous articles, which proved that the most frequent Mafa 170 haplotype in the Filipino macaque population contains two $M H C$ - $A$ and three $M H C-B$ loci ${ }^{34,52}$.

Among the $100 \mathrm{MHC}$ class I alleles, the most frequently shared $M a f a-A$ molecules, containing the same amino acid sequences in exons 2 and 3 with distribution frequency greater than $10 \%$, were Mafa-A1*007:01 (8/30, 26.7\%), Mafa-A1*056:03 (7/30, 23.3\%) and Mafa-A1*040:03 $(4 / 30,13.3 \%)$. Similarly, the most frequently shared Mafa-B alleles with distribution frequency greater than 10\% were Mafa-B*007:01 (12/30, 40\%), Mafa-B*039:01 (8/30, 26.7\%), Mafa-

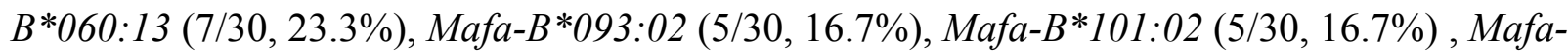
$B * 030: 17(4 / 30,13.3 \%)$, Mafa-B*144:01 (4/30, 13.3\%) and Mafa-B*145:01 (4/30, 13.3\%). A summary of the shared alleles and the number of allele clones identified in each macaque are shown in Figure 1. All of the Mafa-A1*007:01, Mafa- $B^{*} 007: 01: 01$, Mafa- $B * 039: 01$ and Mafa$B^{*} 060: 13$ were detected to express in individuals 4,11 , and 29 , respectively, which indicates that some of them may segregate on one haplotype. Macaque MHC class I allele haplotypes contain variable numbers of loci, which makes them more difficult to characterize than their human counterparts. Although the next-generation sequencing (NGS) techniques have been reported to be effective for high-throughput genotyping of MHC genes and for the detection of low-levelexpressed MHC alleles ${ }^{30,53}$, the new technologies are error-prone because it can be more difficult to discriminate between sequencing errors and true rare alleles. Nevertheless, this problem can be overcome by applying the conventional Sanger sequencing methods. The combined use of the conventional Sanger sequencing methods and the NGS techniques can make the characterization of the highly duplicated macaque $M H C-A /-B$ alleles easier to perform ${ }^{34}$.

\section{Analysis of alleles shared with other populations or with other species}

The 72 known alleles identified in this study were compared with other populations from Filipino, Indonesian, Malaysian and Mauritian origin. We found that the majority of them (57) were reported previously in Vietnamese origin population ${ }^{49-51,54}$, while the remaining 15 alleles 
share the same exons 2 and 3 sequences with other populations. One of the 15 alleles, namely Mafa- $B^{*} 137: 03$, was identical to sequences previously described in both Filipino and Indonesian origin populations $^{28,34}$. For the other 14 alleles, 6 of them were found identical to Indonesianorigin counterparts ${ }^{28,52,55}, 4$ shared with Filipino-origin population ${ }^{34}$ and 4 with Malaysian-origin cynomolgus macaque ${ }^{55}$. None allele was found similar to Mauritian-origin population. Interestingly, two sequences of the Mafa-A4*14 lineage, Mafa-A4*14:03 and Mafa-A4*14:17 identified in this study exhibit identical exon 2 and 3 sequences to $M a m u-A 4 * 14: 03$. Meanwhile, the same exon 2 and 3 sequences were shared with Filipino-origin cynomolgus macaque and Malaysian-origin population ${ }^{56}$ (Table 1), which indicates that this fragment is conserved in macaque during evolution. Surprisingly, the Mamu- $A 4 * 14: 03$ allele was reported to be expressed mainly inside the cell, in contrast to Mamu- $A$-encoded molecules which are mostly found on the cell surface. The different expression patterns were assigned to the antigen-binding $\alpha 1$ and $\alpha 2$ domains ${ }^{57}$. It is possible that the two $M a f a-A 4 * 14$ alleles take the same expression pattern in cynomolgus macaque as those for the Mamu- $A 4 * 14: 03$ and they have some important functions in the cell rather than presenting peptides on the cell surface to T cells. Meanwhile, there are also 5 alleles possessing the same deduced amino acid sequences encoding $\alpha 1$ and $\alpha 2$ domains as their counterparts from other populations, including 2 from Malaysia, 1 from Philippines, 1 from Indonesia and the last one shared with Philippines and Indonesia (Tables 1 and 2). No Mauritian origin sequences were matched.

Hence, in this study, we discovered 15 sequences with perfect identity and 6 sequences with identical amino acid sequences encoding $\alpha 1$ and $\alpha 2$ domains to previously defined MHC class I alleles from Indonesian, Filipino, or Malaysian populations. The sharing of alleles between these geographically distinct populations was consistent with the findings of previous studies, i.e. , there is considerable overlap between different populations for some $M a f a-A$ or $-B$ lineages at the three-digit level of classification, despite the fact that most $M a f a-A$ or $-B$ alleles are population specific $^{52}$. Therefore, the majority of $M a f a-A$ or $-B$ alleles in distinct populations probably finetuned their sequences to cope with environmental pathogens, along with a few parts inherited 
221

222

223

224

225

226

227

228

229

230

231

232

233

234

235

236

237

238

239

240

241

242

243

244

245

246

conservatively. It is believed that these shared alleles between continental (Vietnamese, Malaysian) and insular (Filipino, Indonesian) subgroups had been generated before the migration of cynomolgus macaques across land bridges between continental Asia and islands of Indonesia during the late Pleistocene epoch ${ }^{52}$.

On the other hand, of the 100 alleles identified, 13 Mafa- $A$ and 19 Mafa-B sequences were identical to previously reported alleles from other macaque species (Tables 1 and 2). These included the rhesus macaque, the southern pig-tailed macaque (Macaca nemestrina, Mane), the Northern pig-tailed macaque (Macaca leonina, Malo) and the Assam Macaque (Macaca assamensis, Maas). M.assamensis inhabits the southern region of Yunnan province, China ${ }^{58}$ and this is the first report of a shared allele $(M H C-B * 039: 01)$ expressed in cynomolgus, rhesus and assamensis macaques. Another 3 lineages were also shared among at least three macaque species, such as $M H C-A 1 * 130: 01, M H C-B * 018: 01$ and $M H C-B * 028: 02$. Interestingly, the shared $M H C-B^{*} 039: 01$, was reported in rhesus macaque that it contains a specific B pocket structural motif and has a unique peptide-binding preference consisting of glycine at the second position. This pocket structure was reported in about $6 \%$ of rhesus macaque sequences but absent in human $H L A$ genes ${ }^{59}$. Our data showed for the first time that the unique B pocket structural motif also occurred in Mafa- $B^{*}$ 039:01 of Vietnamese origin with high frequency. The biological significance of this molecule needs to be further analyzed in the future and needs to be concerned when using Vietnamese-origin cynomolgus macaques in biomedical researches.

As presented in tables 1 and 2, five alleles were shared with Indian-origin rhesus macaque. Meanwhile, 19 alleles were shared with Chinese-origin rhesus macaque. It can be easily noticed that cynomolgus macaque shares more alleles with Chinese-origin than with Indian-origin rhesus macaque. This may be explained by the overlap in the geographical areas inhabited by both species in eastern Asia, where the two species are likely to hybridize with extensive ancient introgression from Chinese rhesus macaque into the Vietnamese-origin cynomolgus macaque population, as reported previously ${ }^{60-62}$. This theory, i.e. ancient hybridization and admixture in 
247

248

249

250

251

252

253

254

255

256

257

258

259

260

261

262

263

264

265

266

267

268

269

270

271

272

macaques, can be also used to explain the fact that the Vietnamese-origin cynomolgus macaque shares more alleles with other macaque species than with other cynomolgus macaque populations ${ }^{63}$. Of these shared alleles between cynomolgus and rhesus macaques, some of them have been found to be associated with diseases in rhesus macaques. For example, Mamu-B*001 was reported to be resistant to CIA ${ }^{18}$. Our study showed that the distribution frequency of Mafa$B^{*} 001$ is $10 \%(3 / 30)$. It is better to screen out animals containing this allele before building the CIA models with cynomolgus macaques. The other five alleles, Mafa-A1*001:01, Mafa$A 1 * 065: 04$, Mafa-A3*13:02, Mafa- $B * 008: 01$ and Mafa- $B * 017: 02$ identified in our laboratory 60,62, are homologous genes of Mamu-A1*001:01, Mamu-A1*065:01, Mamu-A3*13:03, Mamu$B * 008: 01$ and $M a m u-B * 017: 01$, which are protective alleles against SIV infection in rhesus macaques. In particular, the distribution frequency of $M a f a-A 1 * 065: 04$ in this cohort is not low $(3 / 30,10 \%)$. This indicates that cynomolgus macaques with these protective alleles may exhibit delayed AIDS progression and longer survival time after SIV infections. The accumulation of cynomolgus macaques carrying these protective alleles is helpful to analyze the virus-host immune interaction and to gain insights into immune protection against the SIV infection ${ }^{64}$.

\section{Analysis of recombination in $M a f a-A$ and $M a f a-B$ alleles}

Recombination event is one of the proposed mechanisms to explain the diversity of MHC alleles. It has been reported that the $M a f a-B^{*} 099$ allele lineage was generated by the recombination of the $M a f a-B * 054$ and the $M a f a-B * 095$ allele lineages in cynomolgus macaque mostly originated from the Philippines ${ }^{65}$. In order to detect the presence of other recombination events in Vietnamese origin cynomolgus macaque, we analyzed 77 Mafa- $A$ and $99 M a f a-B$ sequences discovered in our laboratory, including data in this study and those previously reported ${ }^{49-51}$. Using the RDP program, four possible recombination events in Mafa- $A$ and Mafa-B alleles, as shown in Table S1, were detected by at least four different recombination detection methods. Among them, three recombinants showed lower sequence similarity with their parents hence require further investigation. Only $M a f a-A 1 * 036: 04$ was detected as a potential recombinant by 
273 five recombination detection methods and exhibited over $98 \%$ sequence similarity with the

274 counterparts from its parents. As shown in Figure 2, the Mafa-A1*036:04 and the Mafa-

$275 A 1 * 091: 02$ contain very similar sequences in exon 3, with minor variation of four nucleotides in

276 their 5' regions. However, the exon 2 sequences of these two alleles differ considerably, with 24

277 nucleotides being mismatched. We also found that the exon 2 sequence of Mafa-A1*036:04 is

278 very similar to the $M a f a-A 1 * 057: 01$ allele, with only two nucleotide differences present in their

2795 ' regions. To determine whether the $M a f a-A 1 * 036: 04$ was created by a recombination event, we

280 further conducted analysis using RIP (Figure S1). The result indicates that the Mafa-A1*036:04

281 allele was possibly generated by a crossover event between Mafa-A1*091:02 and Mafa-

$282 A 1 * 057: 01$. The exact breakpoint cannot be defined because their intron sequences are not

283 available in this study. Additionally, we further performed phylogenetic analysis of all reported

284 sequences belonging to the $A 1 * 036, A 1 * 057$ and $A 1 * 091$ lineages from cynomolgus and rhesus

285 macaques. The phylogeny map of exon 2 sequences presented in Figure 3A showed that Mafa-

$286 A 1^{*} 036$ cluster more closely to Mafa- $A 1 * 057$ than the $M a f a-A 1 * 091$. While in the phylogenetic

287 tree of exon 3 presented in Figure 3B, Mafa- $A 1 * 036$ separates $M a f a-A 1 * 057$ in different

288 branches and groups $M a f a-A 1 * 091$ in a cluster. We also found that the $-A 1 * 057$ and $-A 1 * 091$

289 lineages are grouped together in these two trees regardless of the species, while the $-A 1 * 036$

290 lineages are separated according to species. Mamu- $A 1 * 036$ exhibits higher sequence similarity

291 with $M a m u-A 1 * 091$ than with $M a m u-A 1 * 057$. It is possible that the $-A 1 * 036$ allele generated by

292 crossover recombination between $-A 1 * 091$ and $-A 1 * 057$ just occurred recently in cynomolgus

293 macaque, but not yet in rhesus macaque.

\section{Analysis of the diversity in Mafa-A1 locus}

295 We have obtained 77 Mafa- $A$ and 99 Mafa- $B$ sequences in our laboratory, including 67 Mafa-

296 A1. Since Mafa-Al was the highest polymorphic gene compared to other Mafa-A loci and the

297 locus number designation for Mafa-B was not yet clear, here we only analyze the diversity in

298 Mafa-A1 locus of Vietnamese origin. The sequences of the 67 Mafa-A1 exons 2 and 3, 
299

300

301

302

303

304

305

306

307

308

309

310

311

312

313

314

315

316

317

318

319

320

321

322

323

324

325

encoding residues 2-182 of the MHC class I protein, were aligned and a total of 157 nucleotide polymorphic sites (28.8\%) were discovered by DnaSP (Table 3). To distinguish positions presenting two or more nucleotides from sites dominated by one nucleotide, we calculated the incidence for the second-most common nucleotide at each position (Table S2). 97 variable sites were considered highly polymorphic with the incidence greater than $5 \%$, while the remaining 60 with the incidence less than 5\% were considered to exhibit rare variation. Analysis on the variability index of the second-most common amino acid residue showed that 48 out of 181 sites $(26.5 \%)$ were defined as highly polymorphic, where the distribution frequency of the second-most common amino acid is greater than 5\% (Table S3). In comparison, only 70 nucleotide positions and 45 amino acid residues in $H L A-A$ were considered highly polymorphic 2. These data showed that cynomolgus macaque $M a f a-A 1$ exhibit higher polymorphism than human $H L A-A$ and several polymorphic sites are macaque-specific ${ }^{52}$. The $\alpha 1$ and $\alpha 2$ domains of MHC class I glycoproteins contains many functional sites that bind peptide antigens and engage T cell receptors. According to previous studies ${ }^{66}$, the deduced 36 PBS and 26 TBS were determined. Among these binding sites, 8 residues were both involved in the interaction with the peptides and the receptors. The diversity of nucleotide sequences encoding PBS or TBS $(\mathrm{Pi}=0.162)$ in the $M a f a-A 1$ was predominantly higher than that in non-PBS or non-TBS coding regions $(\mathrm{Pi}=0.035)$ of the corresponding alleles (Table 3$)$. This is consistent with the observation that high polymorphism at these functional residues is significant to increase the depth and breadth of the weaponry to cope with variant pathogens during evolution ${ }^{2,67}$. All of these 54 functional residues were listed in Figure 4, including 34 positions with high polymorphism. On the other hand, 16 out of the 54 functional residues are completely conserved in the $67 \mathrm{Mafa}-\mathrm{Al}$ sequences. 8 of the 16 residues are also conserved in human, including Y7, Y59, and Y159. The three tyrosine residues were located at an end of the peptide binding groove and may contribute to the recognition of a constant feature of processed antigens ${ }^{68}$ which indicates that these conserved residues are also important to maintain some constant features for presenting peptide and for lymphocyte recognition during evolution. 
326

327

328

329

330

331

332

333

334

335

336

337

338

339

340

341

342

343

344

345

346

347

348

349

350

351

352

\section{Conclusion}

In this study, we have identified $40 \mathrm{Mafa}-\mathrm{A}$ and $60 \mathrm{Mafa}-\mathrm{B}$ alleles from 30 unrelated cynomolgus macaques of Vietnamese origin. 28 of these alleles were found to be novel ones. Each monkey expressed 1 to 5 Mafa- $A$ genes and 2 to 7 Mafa-B genes. These data showed that both $M H C-A$ and $-B$, especially $-B$ genes, were duplicated in cynomolgus macaque of Vietnamese origin. We also identified some alleles with distribution frequency greater than 10\% and four alleles (Mafa-A1*007:01, Mafa-B*007:01:01, Mafa-B*039:01 and Mafa$B * 060: 13)$ were detected to express simultaneously in three individuals. Whether these four alleles segregate on one haplotype need to be verified in future study. Among the 72 known alleles, 15 alleles share the same exons 2 and 3 sequences with other populations, including Filipino, Indonesian and Malaysian origin populations. The sharing of alleles between these geographically distinct populations indicates that a few alleles preserved conservatively in evolution may exercise vital immune functions, and many of the Mafa-A or $-B$ alleles in distinct populations probably fine-tuned their sequences to cope with environmental pathogens.

On the other hand, 32 sequences were identical to previously reported alleles from other macaque species, including 19 shared with Chinese-origin rhesus macaque. The fact that the Vietnamese-origin cynomolgus macaque shares more alleles with Chinese-origin rhesus macaque than with other cynomolgus macaque populations may be explained by ancient hybridization and admixture in macaques. The five alleles identified in our laboratory are homologous genes of protective factors after SIV challenge in rhesus macaque. In this regard, cynomolgus macaque of Vietnamese origin carrying these protective alleles will be a good alternative model to study the immune protection mechanism of SIV infection. To further explain the diversity of Mafa- $A$ and $-B$ genes, recombination events and the variability in $M a f a-A 1$ locus were analyzed, the Mafa- $A 1 * 036: 04$ allele was possibly generated by a crossover event between Mafa- $A 1 * 091: 02$ and Mafa- $A 1 * 057: 01$, which occurred recently in cynomolgus macaque, but not in rhesus macaque yet. 97 variable nucleotide positions in Mafa$A 1$ exons 2 and 3 sequences and 48 amino acid sites in the $\alpha 1$ and $\alpha 2$ domains were considered 
353 highly polymorphic. In comparison to human, the exons 2 and 3 sequences from cynomolgus

354 macaque exhibit higher polymorphism. The information on the diversity of these MHC class I

355 alleles will facilitate the use of Vietnamese-origin cynomolgus macaques to test the therapeutic

356 efficacy and potential side effects of vaccines or other drugs.

357

358

359 Acknowledgments

360 We are grateful for the Primate Research Center of South China for helping with the cynomolgus

361 macaque blood collection, and for IPD-MHC Nomenclature Committee for providing the

362 designations for the MHC class I alleles.

363 Ethics:

364 The methods of using animals in this study had been reviewed and approved by the institutional

365 Animal Care and Use Committees (IACUC), Guangdong Landau Biotechnology Co. Ltd. The

366 date of approval was Oct.8,2015. The approval code was IACUC-003.

367

368 Funding:

369 This study was funded by the National Natural Science Foundation of China (31401088) and

370 supported by the Fundamental Research Funds for the Central Universities of South China

371 University of Technology (2014ZZ0056).

372

373 Disclosures:

374 There are no conflicts of interest among all authors in this article. 


\section{Reference}

378 1. Parham P. MHC class I molecules and KIRs in human history, health and survival. Nature reviews Immunology. 2005;5(3):201-214.

2. Robinson J, Guethlein LA, Cereb N, et al. Distinguishing functional polymorphism from random variation in the sequences of >10,000 HLA-A, -B and -C alleles. PLoS Genet. 2017;13(6):e1006862.

3. Steiner NK, Baldassarre L, Koester R, Ng J, Hartzman RJ, Hurley CK. Novel HLA-B alleles formed by an inter-locus recombination with HLA-C, HLA-B*0713 and B*6702. Tissue Antigens. 2002;59(2):148-150.

4. Bahr A, Wilson AB. The evolution of MHC diversity: evidence of intralocus gene conversion and recombination in a single-locus system. Gene. 2012;497(1):52-57.

5. Nomura T, Matano T. Association of MHC-I genotypes with disease progression in HIV/SIV infections. Frontiers in Microbiology. 2012;3:234.

6. Kuniholm MH, Xiaojiang G, Xiaonan X, et al. Human leukocyte antigen genotype and risk of HIV disease progression before and after initiation of antiretroviral therapy. Journal of Virology. 2011;85(20):1082610833.

7. Shiina T, Blancher A, Inoko H, Kulski JK. Comparative genomics of the human, macaque and mouse major histocompatibility complex. Immunology. 2016.

8. Lenz TL, Deutsch AJ, Han B, et al. Widespread non-additive and interaction effects within HLA loci modulate the risk of autoimmune diseases. Nat Genet. 2015;47(9):1085-1090.

9. Raychaudhuri S, Sandor C, Stahl EA, et al. Five amino acids in three HLA proteins explain most of the association between MHC and seropositive rheumatoid arthritis. Nat Genet. 2012;44(3):291-296.

10. Martin MP, Mary C. Immunogenetics of HIV disease. Immunological Reviews. 2013;254(1):245-264.

11. Vierboom M, Breedveld E, Kondova I, t Hart BA. The significance of non-human primates as preclinical models of human arthritic disease. Expert Opin Drug Discov. 2008;3(3):299-310.

12. Smits SL, van den Brand JM, de Lang A, et al. Distinct severe acute respiratory syndrome coronavirusinduced acute lung injury pathways in two different nonhuman primate species. $J$ Virol. 2011;85(9):42344245.

13. Lin PL, Rodgers M, Smith L, et al. Quantitative Comparison of Active and Latent Tuberculosis in the Cynomolgus Macaque Model. Infection and Immunity. 2009;77(10):4631-4642.

14. Kisu I, Mihara M, Banno K, et al. Uterus allotransplantation in cynomolgus macaque: a preliminary experience with non-human primate models. J Obstet Gynaecol Res. 2014;40(4):907-918.

15. Walter L, Ansari AA. MHC and KIR Polymorphisms in Rhesus Macaque SIV Infection. Front Immunol. 2015;6:540.

16. Yi WC, Finstad CL, Walfield AM, et al. Site-specific UBITh amyloid-beta vaccine for immunotherapy of Alzheimer's disease. Vaccine. 2007;25(16):3041-3052.

17. Emborg ME. Nonhuman primate models of Parkinson's disease. Ilar Journal. 2007;48(4):339. 
412 18. Bakker NP, Erck MG, Van, Otting N, et al. Resistance to collagen-induced arthritis in a nonhuman primate

413

414

415

416

417

418

419

420

421

422

423

424

425

426

427

428

429

430

431

432

433

434

435

436

437

438

439

440

441

442

443

444

445

446

447

448

449

450

451

452 species maps to the major histocompatibility complex class I region. Journal of Experimental Medicine. 1992;175(4):933-937.

19. Vierboom MP, Breedveld E, Kap YS, et al. Clinical efficacy of a new CD28-targeting antagonist of T cell co-stimulation in a non-human primate model of collagen-induced arthritis. Clinical \& Experimental Immunology. 2016;183(3):405-418.

20. Loffredo JT, Maxwell J, Qi Y, et al. Mamu-B*08-positive macaques control simian immunodeficiency virus replication. J Virol. 2007;81(16):8827-8832.

21. Wambua D, Henderson R, Solomon C, et al. SIV-infected Chinese-origin rhesus macaques express specific MHC class I alleles in either elite controllers or normal progressors. J Med Primatol. 2011;40(4):244-247.

22. Allen TM, Sidney J, ., Guercio MF, Del, et al. Characterization of the peptide binding motif of a rhesus MHC class I molecule (Mamu-A*01) that binds an immunodominant CTL epitope from simian immunodeficiency virus. Journal of Immunology. 1998;160(12):6062-6071.

23. Mothe BR, Sidney J, Dzuris JL, et al. Characterization of the Peptide-Binding Specificity of Mamu-B*17 and Identification of Mamu-B*17-Restricted Epitopes Derived from Simian Immunodeficiency Virus Proteins. The Journal of Immunology. 2002;169(1):210-219.

24. Loffredo JT, Sidney J, Bean AT, et al. Two MHC class I molecules associated with elite control of immunodeficiency virus replication, Mamu-B*08 and HLA-B*2705, bind peptides with sequence similarity. J Immunol. 2009;182(12):7763-7775.

25. Gumert MD. The common monkey of Southeast Asia: long-tailed macaque populations, ethnophoresy, and their occurrence in human environments. Book Chapter. 2011.

26. Sussman RW, Tattersall I. Distribution, abundance, and putative ecological strategy of Macaca fascicularis on the island of Mauritius, southwestern Indian Ocean. Folia primatologica. 1986;46 (1):28-43.

27. Otting N, de Vos-Rouweler AJ, Heijmans CM, de Groot NG, Doxiadis GG, Bontrop RE. MHC class I A region diversity and polymorphism in macaque species. Immunogenetics. 2007;59(5):367-375.

28. Pendley CJ, Becker EA, Karl JA, et al. MHC class I characterization of Indonesian cynomolgus macaques. Immunogenetics. 2008;60(7):339-351.

29. Campbell KJ, Detmer AM, Karl JA, et al. Characterization of $47 \mathrm{MHC}$ class I sequences in Filipino cynomolgus macaques. Immunogenetics. 2009;61(3):177-187.

30. Budde ML, Wiseman RW, Karl JA, Hanczaruk B, Simen BB, O'Connor DH. Characterization of Mauritian cynomolgus macaque major histocompatibility complex class I haplotypes by high-resolution pyrosequencing. Immunogenetics. 2010;62(11-12):773-780.

31. Seekatz AM, Aruna P, Rasko DA, et al. Differential response of the cynomolgus macaque gut microbiota to Shigella infection. PloS one. 2013;8(6):e64212.

32. Robinson J, Mistry K, Mcwilliam H, Lopez R, Parham P, Marsh SGE. The IMGT/HLA database. Methods in Molecular Biology. 2011;39(Database issue):1171-1176.

33. Shiina T, Tamiya G, Oka A, Takishima N, Inoko H. Genome sequencing analysis of the $1.8 \mathrm{Mb}$ entire human MHC class I region. Immunological Reviews. 1999;167(1):193-199.

34. Shiina T, Yamada Y, Aarnink A, et al. Discovery of novel MHC-class I alleles and haplotypes in Filipino cynomolgus macaques (Macaca fascicularis) by pyrosequencing and Sanger sequencing: Mafa-class I polymorphism. Immunogenetics. 2015;67(10):563-578.

Peer) reviewing PDF | (2019:05:37873:1:0:NEW 2 Sep 2019) 
453 35. Wiseman RW, Karl JA, Bohn PS, Nimityongskul FA, Starrett GJ, O'Connor DH. Haplessly hoping: macaque major histocompatibility complex made easy. ILAR J. 2013;54(2):196-210.

36. Maccari G, Robinson J, Ballingall K, et al. IPD-MHC 2.0: an improved inter-species database for the study of the major histocompatibility complex. Nucleic acids research. 2016;45(D1):D860-D864.

37. Karl JA, Graham ME, Wiseman RW, et al. Major histocompatibility complex haplotyping and long-amplicon allele discovery in cynomolgus macaques from Chinese breeding facilities. Immunogenetics. 2017:1-19.

38. Burland TG. DNASTAR's Lasergene sequence analysis software. Methods in Molecular Biology. 2000;132:71-91.

39. Hall BTA. BioEdit: a user-friendly biological sequence alignment editor and analysis program for windows 95/98. Paper presented at: Nucl. Acids. Symp. Ser2015.

40. Robinson J, Halliwell JA, McWilliam H, Lopez R, Marsh SG. IPD-the Immuno Polymorphism Database. Nucleic Acids Res. 2013;41(Database issue):D1234-1240.

41. de Groot NG, Otting N, Robinson J, et al. Nomenclature report on the major histocompatibility complex genes and alleles of Great Ape, Old and New World monkey species. Immunogenetics. 2012;64(8):615-631.

42. Martin DP, Murrell B, Golden M, Khoosal A, Muhire B. RDP4: Detection and analysis of recombination patterns in virus genomes. Virus Evol. 2015;1(1):vev003.

43. Zhao M, Wang Y, Shen $\mathrm{H}$, et al. Evolution by selection, recombination, and gene duplication in MHC class I genes of two Rhacophoridae species. Bmc Evolutionary Biology. 2013;13(1):1-13.

44. Saitou N, Nei M. The neighbor-joining method: a new method for reconstructing phylogenetic trees. Molbiolevol. 1987;4(6):406-425.

45. Kumar S, Stecher G, Tamura K. MEGA7: Molecular Evolutionary Genetics Analysis Version 7.0 for Bigger Datasets. Mol Biol Evol. 2016;33(7):1870-1874.

46. Kimura M. A simple method for estimating evolutionary rate of base substitutions through comparative studies of nucleotide sequences. Journal of Molecular Evolution. 1981;16(2):111-120.

47. Peer E, Rechavi G, Dan D. Epitranscriptomics: regulation of mRNA metabolism through modifications. Current Opinion in Chemical Biology. 2017;41:93-98.

48. Grozhik AV, Jaffrey SR. Epitranscriptomics: Shrinking maps of RNA modifications. Nature. 2017;551(7679):174-176.

49. Zhang GQ, Ni C, Ling F, et al. Characterization of the major histocompatibility complex class I A alleles in cynomolgus macaques of Vietnamese origin. Tissue Antigens. 2012;80(6):494-501.

50. Wang HB, Ling F, Zhuo M, Wang JF, Wang XN. Eighteen novel MHC class I A alleles identified in Vietnamese-origin cynomolgus macaques. Tissue Antigens. 2011;78(2):139-142.

51. Wang HB, Ling F, Zhuo M, Wang JF, Wang XN. Twenty-three novel major histocompatibility complex class I B alleles identified in cynomolgus macaques of Vietnamese origin. Tissue Antigens. 2011;77(4):346348.

52. Kita YF, Hosomichi K, Kohara S, et al. MHC class I A loci polymorphism and diversity in three Southeast Asian populations of cynomolgus macaque. Immunogenetics. 2009;61(9):635-648.

53. Wiseman RW, Karl JA, Bimber BN, et al. Major histocompatibility complex genotyping with massively parallel pyrosequencing. Nat Med. 2009;15(11):1322-1326.

54. Krebs KC, Jin Z, Rudersdorf R, Hughes AL, O'Connor DH. Unusually High Frequency MHC Class I Alleles in Mauritian Origin Cynomolgus Macaques. The Journal of Immunology. 2005;175(8):5230-5239.

Peer] reviewing PDF | (2019:05:37873:1:0:NEW 2 Sep 2019) 
494 55. Saito Y, Naruse TK, Akari H, Matano T, Kimura A. Diversity of MHC class I haplotypes in cynomolgus

495

496

497

498

499

500

501

502

503

504

505

506

507

508

509

510

511

512

513

514

515

516

517

518

519

520

521

522

523

524

525

526

527 macaques. Immunogenetics. 2012;64(2):131-141.

56. Aarnink A, Apoil PA, Takahashi I, Osada N, Blancher A. Characterization of MHC class I transcripts of a Malaysian cynomolgus macaque by high-throughput pyrosequencing and EST libraries. Immunogenetics. 2011;63(11):703-713.

57. Rosner C, Kruse PH, Lubke T, Walter L. Rhesus macaque MHC class I molecules show differential subcellular localizations. Immunogenetics. 2010;62(3):149-158.

58. Yan X, Li A, Zeng L, et al. Identification of MHC class I sequences in four species of Macaca of China. Immunogenetics. 2013;65(12):851-859.

59. Sette A, Sidney J, Southwood S, et al. A shared MHC supertype motif emerges by convergent evolution in macaques and mice, but is totally absent in human MHC molecules. Immunogenetics. 2012;64(6):421-434.

60. Bonhomme M, Cuartero S, Blancher A, Crouauroy B. Assessing Natural Introgression in 2 Biomedical Model Species, the Rhesus Macaque (Macaca mulatta) and the Long-Tailed Macaque (Macaca fascicularis). Journal of Heredity. 2009;100(2):158.

61. Stevison LS, Kohn MH. Divergence population genetic analysis of hybridization between rhesus and cynomolgus macaques. Molecular Ecology. 2009;18(11):2457-2475.

62. Kanthaswamy S, Satkoski J, George D, Kou A, Erickson BJ, Smith DG. Interspecies Hybridization and the Stratification of Nuclear Genetic Variation of Rhesus (Macaca Mulatta) and Long-Tailed Macaques (Macaca Fascicularis). Int J Primatol. 2008;29(5):1295-1311.

63. Fan Z, Zhou A, Osada N, et al. Ancient hybridization and admixture in macaques (genus Macaca) inferred from whole genome sequences. Molecular Phylogenetics \& Evolution. 2018:S105579031730489X.

64. Mudd PA, Martins MA, Ericsen AJ, et al. Vaccine-induced CD8+ T cells control AIDS virus replication. Nature. 2012;491(7422):129-133.

65. Orysiuk D, Lawrence J, Prashar T, et al. Evidence of recombination producing allelic diversity in MHC class I Mafa-B and -A alleles in cynomolgus macaques. Tissue Antigens. 2012;79(5):351-358.

66. Lafont BAP, Buckler-White A, Plishka R, Buckler C, Martin MA. Characterization of Pig-Tailed Macaque Classical MHC Class I Genes: Implications for MHC Evolution and Antigen Presentation in Macaques. The Journal of Immunology. 2003;171(2):875-885.

67. Lian XD, Zhang XH, Dai ZX, Zheng YT. Cloning, sequencing, and polymorphism analysis of novel classical MHC class I alleles in northern pig-tailed macaques ( Macaca leonina ). Immunogenetics. 2016;68(4):261274.

68. Bjorkman PJ, Saper MA, Samraoui B, Bennett WS, Strominger JL, Wiley DC. The foreign antigen binding site and T cell recognition regions of class I histocompatibility antigens. Nature. 1987;329(6139):512-518. 
Figure $\mathbf{1}$ (on next page)

Figure 1. Summary of MHC class $I-A$ and $-B$ alleles identified from 30 cynomolgusmacaques of Vietnamese origin. 
Figure 1

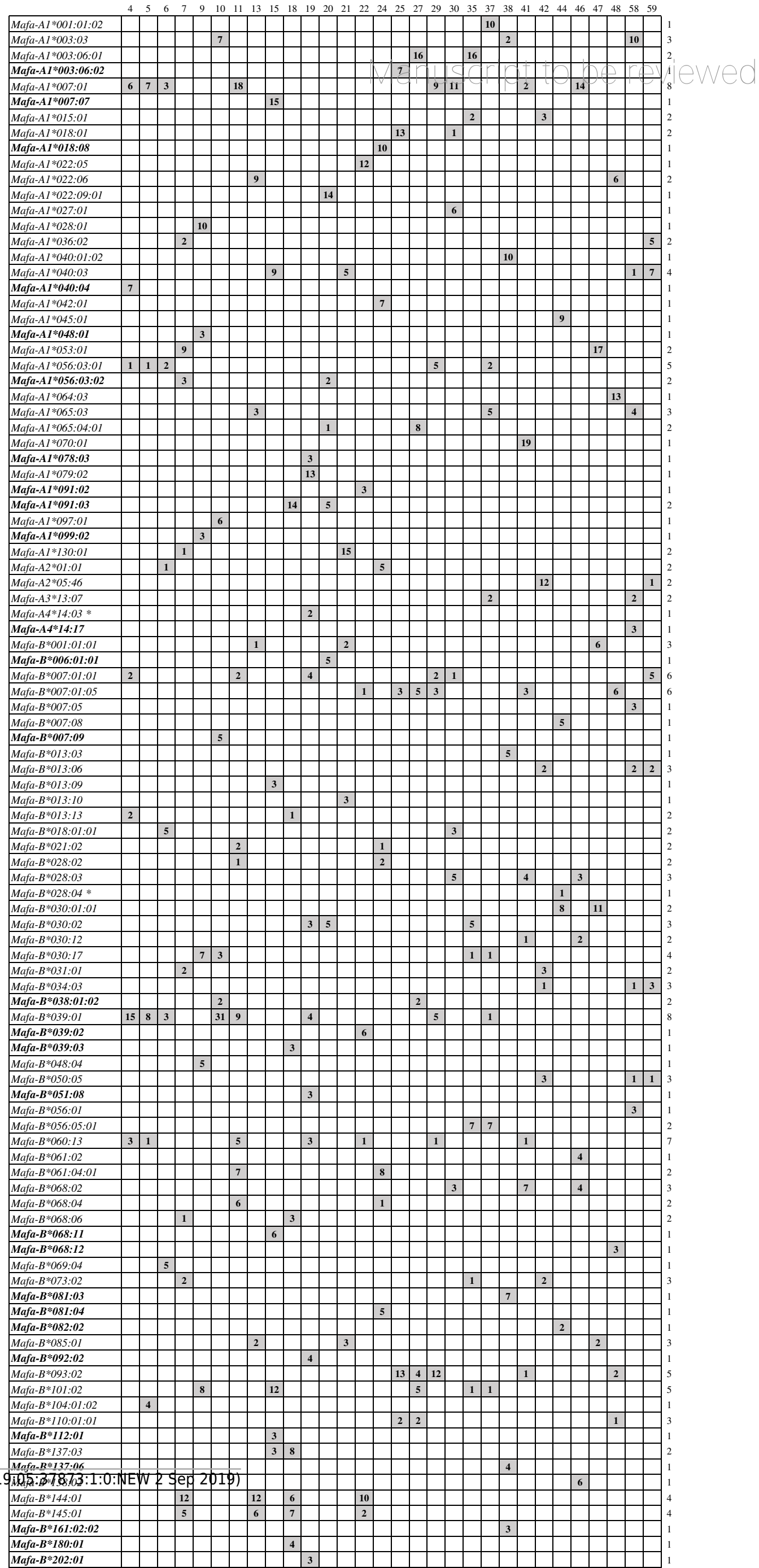


Figure 1. Summary of MHC class $I-A$ and $-B$ alleles identified from 30 cynomolgus macaques of Vietnamese origin. The novel alleles are represented in bold. The number of cDNA clones for every allele detected in each animal are shown in the cells and highlighted in grey. The numbers of individuals sharing the particular allele are shown at the end of each line. The two asterisk-labeled alleles (Mafa- $A 4 * 14: 03$ and Mafa- $B^{* 028: 04)}$ are also identified in other individuals which are not in these 30 animals, respectively. 
Figure 2

Figure 2. The nucleotide sequences alignment of Mafa-A1*036:04,Mafa-A1*057:01 and Mafa-A1*091:02. 


\section{Figure 2}

Exon 2

Mafa-A1*036:04 GGCTCCCACT CCATGAGGTA TTTCTACACC TCCATGTCCC GGCCCGGCCG CGGGCAGCCC CGCTTCATCG CCGTGGGCTA CGTGGACGAC ACGCAGTTC Mafa-A1*057:01 Mafa-A1*091:02

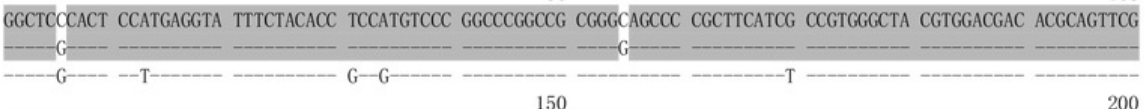

Mafa-A1*036:04 TGCGGTTCGA CAGCGACGCC GCGAGCCAAA GGATGGAGCC GCGGGCGCCG TGGGTGGAGC AGGAGGGTCC AGAGTATTGG GACTCGCAGA CACGGATCAT

Mafa-A1*057:01

Mafa-A1*091:02

Mafa-A1*036:04 GAAGGCCGAC ACACAGACCT ACCGAGAGAG CCTGCGGAAC CTGCGCGGCT ACTACAACCA GAGCGAGGCC

Mafa-A1*057:01

Mafa-A1*091:02

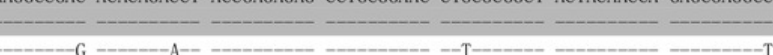

Exon 3

320

370

Mafa-A1*036:04 GGGTCTCACA CCATCCAGTG GATGTACGGC TGCGACCTGG GGCCCGACGG GCGCCTCCTC CGCGGGTATG ACCAGTCCGC CTACGACGGC AGGGATTACA

Mafa-A1*057:01
Mafa-A1*091:02

Mafa-A1*091:02

Mafa-A1*036:04 TCGCCCTGAA CGAGGACCTG CGCTCCTGGA CCGCCGCGGA CATGGCGGCT CAGAACACCC AGCGGAAGTG GGAGGCGGCG GGTGCGGCGG AGCAGATGAG

Mafa-A1*057:01 --T-

Mafa-A1*091:02

Mafa-A1*036:04 AGCCTACCTG GAGGGCGAGT GCCTGGAGTG GCTCCGCAGA TACCTGGAGA ACGGGAAGGA GACGCTGCAG CGCGCG

Mafa-A1*057:01

Mafa-A1*091:02

546

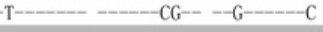

$-\mathrm{CG}--\mathrm{G}-\cdots-\mathrm{C}-\cdots-\cdots$

Figure 2. The nucleotide sequences alignment of Mafa-A1*036:04, Mafa-A1*057:01 and $M a f a-A 1 * 091: 02$. Nucleotide identical to the top sequence is indicated by a hyphen. Nucleotide numbers are shown above. $M a f a-A 1 * 036: 04$ appears to be a result of a crossover between Mafa-A1*057:01 (exon 2, grey marked) and Mafa-A1*091:02 (exon 3, grey marked). 
Figure 3

Figure 3. Phylogenetic analysisof exon 2 (A) and exon 3 (B) of $A 1 * 036, A 1 * 57$ and $A 1 * 091$ alleles from cynomolgus and rhesusmacaques. 
Figure 3

(A)

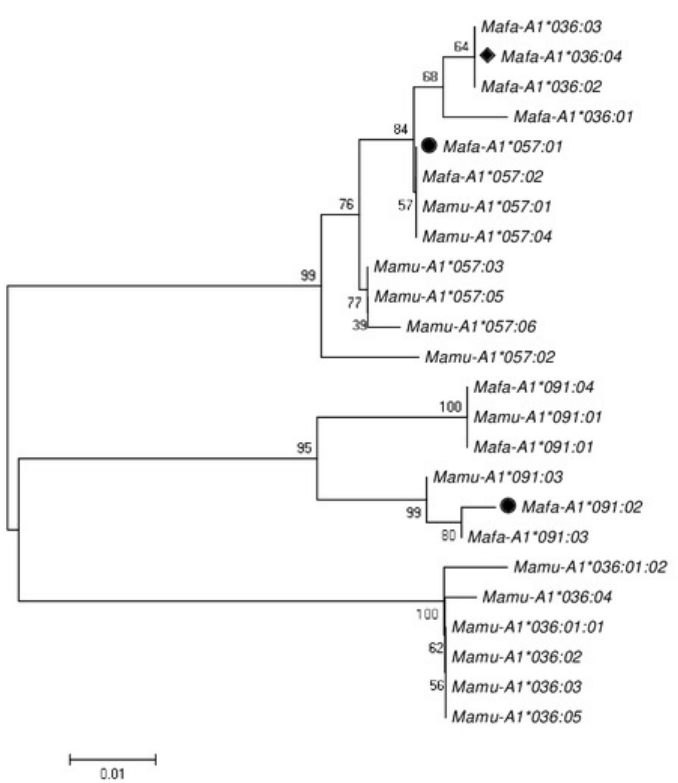

(B)

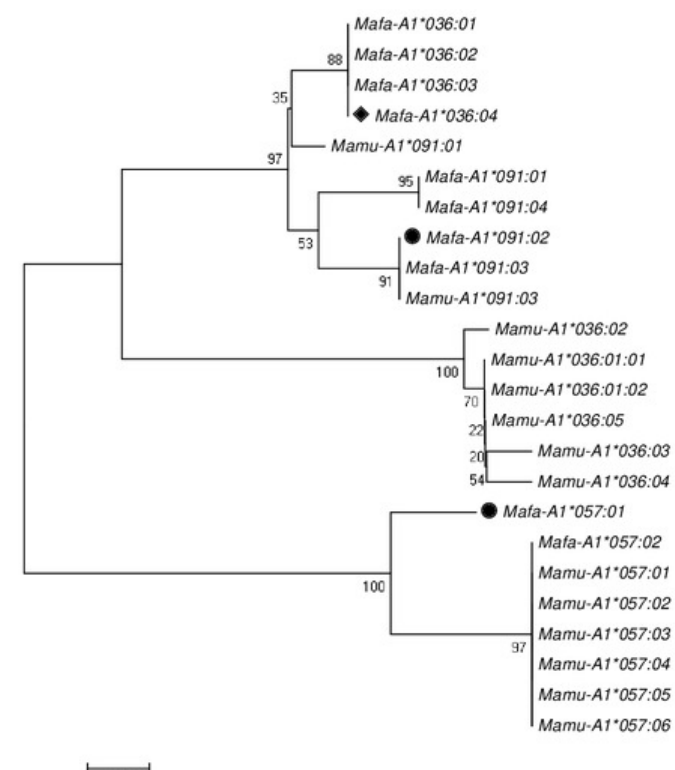

Figure 3. Phylogenetic analysis of exon 2 (A) and exon 3 (B) of $A 1 * 036, A 1 * 57$ and $A 1 * 091$ alleles from cynomolgus and rhesus macaques. Solid circles represent alleles for recombinant parents and the solid diamonds indicate the recombinant. The numbers at branches indicate bootstrap values. 
Figure 4

Figure 4. The diversity of amino acid residues at 54 functional positions relativeto PBS or TBS in 67 Mafa-A1 ofVietnamese origin. 
Figure 4
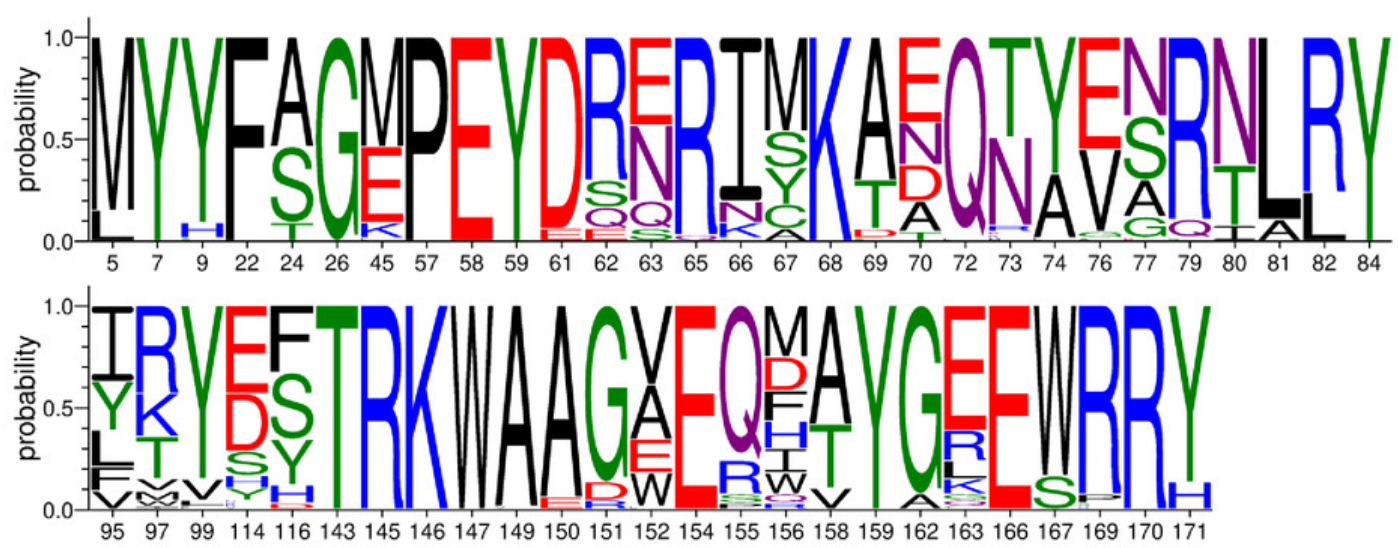

Figure 4. The diversity of amino acid residues at 54 functional positions relative to PBS or TBS in 67 $M a f a-A I$ of Vietnamese origin. The height of symbols within the stack reflects the distribution frequency of each amino acid residue. The 54 functional residues were determined according to Ref.51 and listed as follows: 28 PBS $(5,7,9,22,24,26,45,59,63,66,67,70,73,74,77,80,81,84,95,97$, $99,114,116,143,147,156,159,171), 18$ TBS $(57,61,65,68,69,72,76,79,82,145,149,150,151$, $154,158,162,166,169)$ and 8 both PBS and TBS $(58,62,146,152,155,163,167,170)$. 


\section{Table $\mathbf{1}$ (on next page)}

Table1 40 Mafa-A alleles detected inVietnamese-origin cynomolgus macaques 
1 Table1 40 Mafa- $A$ alleles detected in Vietnamese-origin cynomolgus macaques

\begin{tabular}{|c|c|c|c|}
\hline Allele name & $\begin{array}{l}\text { Accession } \\
\text { number }\end{array}$ & Other origin & Macaque counterparts ${ }^{\text {a }}$ \\
\hline Mafa-A1*001:01:02 & KT907313 & $A 1 * 001: 01: 01(\mathrm{MacM})^{\mathrm{c}}$ & Mamu-A1*001:01(U50836-I) \\
\hline$M a f a-A 1 * 003: 03$ & KT907312 & & Maтu-A1*003:01:01(U41379-Unk) \\
\hline$M a f a-A 1 * 003: 06: 01$ & KT907326 & & \\
\hline$\underline{M a f a-A 1 * 003: 06: 02}$ & KT907327 & & \\
\hline$M a f a-A 1 * 007: 01$ & KT907328 & & \\
\hline$\underline{M a f a-A 1 * 007: 07}$ & KT907316 & & Мати-A1*007:02(AF157397-Unk) \\
\hline Mafa-A1*015:01 & KT907351 & & Mamu-A1*015:01(AB551785-Bu) \\
\hline Mafa-A1*018:01 & KT907329 & & \\
\hline$\underline{\text { Mafa- } A 1 * 018: 08}$ & KT907330 & & \\
\hline$M a f a-A 1 * 022: 05$ & KT907331 & & \\
\hline$M a f a-A 1 * 022: 06$ & KT907309 & & \\
\hline$M a f a-A 1 * 022: 09: 01$ & KT907332 & & \\
\hline Mafa-A1*027:01 & KT907333 & & \\
\hline$M a f a-A 1 * 028: 01$ & KT907334 & & \\
\hline Mafa-A1*036:02 & KY073130 & & \\
\hline Mafa- $A 1 * 040: 01: 02$ & KT907315 & & \\
\hline Mafa-A1*040:03 & KT907321 & & \\
\hline$\underline{\text { Mafa }-A 1 * 040: 04}$ & KT907322 & & \\
\hline Mafa-A1*042:01 & KT907324 & & \\
\hline$M a f a-A 1 * 045: 01$ & KT907335 & & Мamu-A1*045:01(EU262741-Ch) \\
\hline$\underline{M a f a-A 1 * 048: 01}$ & KT907348 & & \\
\hline Mafa- $A 1 * 053: 01$ & KT907336 & & Mamu-A1*053:02(EU551177-Ch) \\
\hline Mafa-A1*056:03:01 & KT907337 & & Mamu-A1*056:02:01(AM295922-Ch) \\
\hline$\underline{M a f a-A 1 * 056: 03: 02}$ & KT907338 & & \\
\hline$M a f a-A 1 * 064: 03$ & KT907325 & & \\
\hline$M a f a-A 1 * 065: 03$ & KT907339 & & \\
\hline$M a f a-A 1 * 065: 04: 01$ & KT907340 & & Mamu-A1*065:01(AB430441-Bu, EU418506-Ch) \\
\hline Mafa-A1*070:01 & KT907341 & $\mathrm{ICM}^{\mathrm{b}}$ & \\
\hline$\underline{\text { Mafa }-A 1 * 078: 03}$ & KT907344 & & \\
\hline$M a f a-A 1 * 079: 02$ & KT907342 & $\mathrm{ICM}^{\mathrm{b}}$ & \\
\hline$\underline{\text { Mafa- } A 1 * 091: 02}$ & KT907319 & & \\
\hline$\underline{M a f a-A 1 * 091: 03}$ & KT907320 & & \\
\hline$M a f a-A 1 * 097: 01$ & KT907318 & $\mathrm{ICM}^{\mathrm{b}}$ & Mamu-A1*109:01(AB444902-Bu) \\
\hline$\underline{\text { Mafa-A1*099:02 }}$ & KT907323 & & \\
\hline \multirow{2}{*}{ Mafa-A1*130:01 } & \multirow{2}{*}{ KT907343 } & & Mane-A1*130:01(LN875412-Unk), \\
\hline & & & Мати-A1*130:01(HG813262-Unk) \\
\hline Mafa-A2*01:01 & KT907314 & & Мати-A2*01:03(AB444917-Bu, GQ902066-Ch) \\
\hline
\end{tabular}




\begin{tabular}{llll}
\hline Mafa- $A 2 * 05: 46$ & KT907345 & & Mamu-A2*05:21(AM295935-Ch) \\
Mafa-A3*13:07 & KT907347 & & \\
Mafa-A4*14:03 & KT907349 & PCM $^{\text {b }}$ & Mamu-A4*14:03:01(AB444876-Bu/I, GU080236-Ch) \\
Mafa-A4*14:17 & KT907350 & $A^{*} 14: 04(\mathrm{MaCM}){ }^{\text {b }}$ & \\
\hline
\end{tabular}

2 The 40 Mafa- $A$ alleles identified from Vietnamese-origin cynomolgus monkeys are listed. The bold and 3 underlined ones indicate newly identified alleles. IPD name, GenBank accession number, other origin and 4 counterpart(s) in other macaque species are listed for each allele.

5 a For alleles shared with other macaque species, the names of their counterparts, accession numbers, as well as 6 regional populations are also listed. I, Indian rhesus macaque; $\mathrm{Bu}$, Burmese rhesus macaque; $\mathrm{Ch}$, Chinese 7 rhesus macaque; Unk, Unknown-origin rhesus macaque.

8 b For alleles shared identical exons 2 and 3 nucleotide sequences with other populations, ICM: Indonesian origin; 9 PCM: filipino origin; MaCM: Malaysian origin.

$10{ }^{\mathrm{c}}$ For alleles shared identical deduced amino acid sequences encoding $\alpha 1$ and $\alpha 2$ domains with other populations, 11 ICM: Indonesian origin; PCM: filipino origin; MaCM: Malaysian origin. 


\section{Table 2 (on next page)}

Table2 60 Mafa-B alleles detected in Vietnamese-origin cynomolgus macaques 
1 Table2 60 Mafa-B alleles detected in Vietnamese-origin cynomolgus macaques

\begin{tabular}{|c|c|c|c|}
\hline Allele name & $\begin{array}{l}\text { Accession } \\
\text { number }\end{array}$ & Other origin & Macaque counterparts ${ }^{a}$ \\
\hline Mafa-B*001:01:01 & KT895485 & & Мати- $B * 001: 01: 01(\mathrm{AB} 477408-\mathrm{Bu}, \mathrm{U} 42837-\mathrm{I})$ \\
\hline Mafa-B*006:01:01 & KT895494 & & Мати $B$ *006:01(U41828-Unk) \\
\hline Mafa- $B * 007: 01: 01$ & KT895444 & $\mathrm{PCM}^{\mathrm{b}}$ & $\begin{array}{l}\text { Мати- } B^{*} 007: 03(\mathrm{AB} 477412-\mathrm{Bu}, \mathrm{EU} 682528-\mathrm{Ch}, \\
\text { AJ556876-I) }\end{array}$ \\
\hline Mafa- $B * 007: 01: 05$ & KT895442 & & \\
\hline Mafa- $B * 007: 05$ & KT895443 & & \\
\hline Mafa- $B * 007: 08$ & KT895446 & & Мати- $B * 007: 04: 01(\mathrm{GQ} 902078-\mathrm{Ch})$ \\
\hline$\underline{M a f a-B} \times \mathbf{0 0 7 : 0 9}$ & KT895445 & & \\
\hline Mafa- $B * 013: 03$ & KT895451 & & \\
\hline Mafa- $B * 013: 06$ & KT895447 & & \\
\hline Mafa- $B * 013: 09$ & KT895448 & $B^{*} 013: 08(\mathrm{PCM} \& \mathrm{ICM})^{\mathrm{c}}$ & \\
\hline Mafa- $B * 013: 10$ & KT895449 & & \\
\hline Mafa- $B^{*} 013: 13$ & KT895450 & & \\
\hline Mafa- $B^{*} 018: 01: 01$ & KT895490 & $\mathrm{ICM}^{\mathrm{b}}$ & 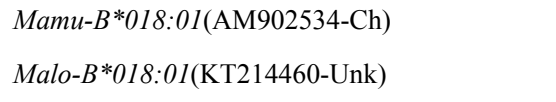 \\
\hline Mafa- $B * 021: 02$ & KT895452 & & Мати- $B^{*} 021: 02(\mathrm{AM} 902536-\mathrm{Bu} / \mathrm{Ch})$ \\
\hline Mafa- $B * 028: 02$ & KT895487 & & $\begin{array}{l}\text { Mane- } B^{*} 028: 01 \text { (FJ875264.1-Unk) } \\
\text { Mamu- } B^{*} 028: 02: 01 \text { (AM902532.1-Ch) }\end{array}$ \\
\hline$M a f a-B * 028: 03$ & KT895486 & $\mathrm{PCM}^{\mathrm{b}}$ & \\
\hline Mafa- $B * 028: 04$ & KY131948 & $\mathrm{ICM}^{\mathrm{b}}$ & \\
\hline Mafa- $B * 030: 01: 01$ & KT895454 & & Мати- $B * 030: 03: 02(\mathrm{AM} 902546-\mathrm{Ch})$ \\
\hline Mafa- $B * 030: 02$ & KT895489 & $\mathrm{MaCM}^{\mathrm{b}}$ & Мати- $B * 030: 03: 03(\mathrm{AM} 902547-\mathrm{Ch})$ \\
\hline Mafa- $B * 030: 12$ & KT895438 & & Mane- $B * 030: 04$ (FJ875259-Unk) \\
\hline Mafa- $B * 030: 17$ & KT895453 & & \\
\hline Mafa- $B * 031: 01$ & KT895491 & & \\
\hline Mafa- $B * 034: 03$ & KT895455 & & \\
\hline$\underline{M a f a-B * 038: 01: 02}$ & KT895456 & & Мати- $B^{*} 038: 02(\mathrm{AB} 477391-\mathrm{Bu})$ \\
\hline Mafa- $B * 039: 01$ & KT895457 & & $\begin{array}{l}\text { Maas- } B * 039: 01(\mathrm{KF} 012951-\mathrm{Ch}) \\
\text { Mamu- } B * 039: 01(\mathrm{AB} 477411-\mathrm{Bu}, \mathrm{EF} 580146-\mathrm{Ch}, \\
\text { AJ556890-I) }\end{array}$ \\
\hline$\underline{M a f a-B * 039: 02}$ & KT895436 & & \\
\hline$\underline{M a f a-B} B \times 039: 03$ & KT895437 & & \\
\hline Mafa- $B * 048: 04$ & KT895458 & & \\
\hline Mafa- $B * 050: 05$ & KT895459 & & \\
\hline$\underline{M a f a-B * 051: 08}$ & KT895460 & & \\
\hline Mafa- $B * 056: 01$ & KT895488 & $\mathrm{ICM}^{\mathrm{b}}$ & Мати- $B$ *056:01(GQ902079-Ch) \\
\hline Mafa- $B * 056: 05: 01$ & KT895461 & & \\
\hline Mafa- $B * 060: 13$ & KT895462 & & \\
\hline
\end{tabular}




\begin{tabular}{|c|c|c|c|}
\hline Mafa- $B^{*} 061: 02$ & KT895464 & $\mathrm{MaCM}^{\mathrm{b}}$ & \\
\hline Mafa- $B^{*} 061: 04: 01$ & KT895463 & & Мати- $B * 061: 02(\mathrm{AM} 902564-\mathrm{Bu} / \mathrm{Ch})$ \\
\hline Mafa- $B^{*} 068: 02$ & KT895466 & & \\
\hline Mafa- $B * 068: 04$ & KT895468 & $\mathrm{MaCM}^{\mathrm{b}}$ & Мати- $B * 068: 04(\mathrm{AM} 902571-\mathrm{Bu} / \mathrm{Ch})$ \\
\hline Mafa- $B^{*} 068: 06$ & KT895467 & & Мати- $B * 068: 02(\mathrm{EF} 219482-$ Unk) \\
\hline$\underline{M a f a-B * 068: 11}$ & KT895465 & & \\
\hline$\underline{M a f a-B * 068: 12}$ & KT895469 & $B^{*} 068: 08(\mathrm{PCM})^{\mathrm{c}}$ & \\
\hline Mafa- $B^{*} 069: 04$ & KT895470 & & \\
\hline Mafa- $B^{*} 073: 02$ & KT895472 & & Мати- ${ }^{*} 073: 01(\mathrm{AB} 477404-\mathrm{Bu}, \mathrm{AM} 902578-\mathrm{Ch})$ \\
\hline$\underline{M a f a-B * 081: 03}$ & KT895473 & & \\
\hline$\underline{M a f a-B * 081: 04}$ & KT895474 & $B^{*} 081: 01(\mathrm{ICM})^{\mathrm{c}}$ & \\
\hline$\underline{M a f a-B * 082: 02}$ & KT895495 & & \\
\hline Mafa- $B^{*} 085: 01$ & KT895484 & $\mathrm{PCM}^{\mathrm{b}}$ & \\
\hline$\underline{M a f a-B * 092: 02}$ & KT895471 & $B^{*} 092: 01(\mathrm{MaCM})^{\mathrm{c}}$ & Мати- $B^{*} 092: 02(\mathrm{AB} 477386-\mathrm{Bu})$ \\
\hline Mafa- $B^{*} 093: 02$ & KT895476 & & \\
\hline Mafa- $B * 101: 02$ & KT895493 & & \\
\hline Mafa- $B * 104: 01: 02$ & KT895477 & & Mane- $B^{*} 104: 02$ (FJ875231-Unk) \\
\hline Mafa- $B * 110: 01: 01$ & KT895478 & & \\
\hline$\underline{M a f a-B * 112: 01}$ & KT895480 & & \\
\hline Mafa- $B^{*} 137: 03$ & KT895439 & PCM, $\mathrm{ICM}^{\mathrm{b}}$ & \\
\hline$\underline{M a f a-B * 137: 06}$ & KT895440 & & \\
\hline Mafa- $B^{*} 138: 02$ & KT895479 & $\mathrm{MaCM}^{\mathrm{b}}$ & \\
\hline Mafa- $B * 144: 01$ & KT895482 & & \\
\hline Mafa- $B * 145: 01$ & KT895483 & & \\
\hline Mafa-B*161:02:02 & KT895481 & & \\
\hline$\underline{M a f a-B * 180: 01}$ & KT895475 & & \\
\hline Mafa-B*202:01 & KT895441 & & \\
\hline
\end{tabular}

2 The 60 Mafa-B alleles identified from Vietnamese-origin cynomolgus monkeys are listed. The bold and underlined ones indicate newly identified 3 alleles. IPD name, GenBank accession number, other origin and counterpart(s) in other macaque species are listed for each allele.

4 a For alleles shared with other macaques species, the name of their counterparts, the accession numbers, as well as regional populations are also

5 listed. I, Indian rhesus macaque; $\mathrm{Bu}$, Burmese rhesus macaque; $\mathrm{Ch}$, Chinese rhesus macaque; Unk, Unknown-origin rhesus macaque.

6 bor alleles shared identical exons 2 and 3 nucleotide sequences with other populations, ICM: Indonesian origin; PCM: filipino origin; MaCM:

7 Malaysian origin.

$8{ }^{\mathrm{c}}$ For alleles shared identical deduced amino acid sequences encoding $\alpha 1$ and $\alpha 2$ domains with other populations, ICM: Indonesian origin; PCM:

9 filipino origin; MaCM: Malaysian origin.

10 


\section{Table 3 (on next page)}

Table 3 Polymorphism of exons 2 and 3 sequences for Mafa-Al of Vietnamese origin 
1 Table 3 Polymorphism of exons 2 and 3 sequences for $M a f a-A 1$ of Vietnamese origin

\begin{tabular}{lccccc}
\hline \multicolumn{1}{c}{ Site } & Ns & S & N & Pi & K \\
\hline All & 546 & 157 & 218 & 0.073 & 39.886 \\
PBS or TBS & 162 & 81 & 127 & 0.162 & 26.316 \\
Non-PBS or TBS & 384 & 76 & 91 & 0.035 & 13.570
\end{tabular}

2

$3 \mathrm{Ns}=$ the number of nucleotides, $\mathrm{S}=$ the number of polymorphic sites, $\mathrm{N}=$ the number of mutations, $\mathrm{Pi}=$ the nucleotide 4 diversity, $\mathrm{K}=$ the average number of nucleotide differences

5

6 